\title{
Blood pressure changes during pregnancy
}

\author{
Hypertension Research (2012) 35, 563-564; doi:10.1038/hr.2012.33; published online 22 March 2012
}

We read, with great interest, the article by Jwa et al. analyzing whether a shift in blood pressure (BP) class, as defined by the Japanese Society of Hypertension Guidelines for the management of hypertension 2009 (JSH 2009), predicts pregnancy-induced hypertension (PIH) among pregnant women in Japan. ${ }^{1}$ The study showed that there was less risk of PIH occurring among pregnant women with low BP at 20 weeks of pregnancy even when these women were found to have high BP at 16 weeks. This interesting finding might indicate that a decreasing $\mathrm{BP}$ in the second trimester (mid-pregnancy fall) has an impact on the occurrence of PIH. We have previously shown that BP measured at home (home BP) falls from the first trimester to the second trimester and then increases up until the time of delivery. ${ }^{2}$ The mid-pregnancy fall in healthy pregnant women might be caused by a decrease in total peripheral vascular resistance owing to vasodilatation in early pregnancy. ${ }^{3}$ The lack of a mid-pregnancy fall among women with pre-eclampsia has suggested a failure of normal cardiovascular adaptation to pregnancy as a result of endothelial dysfunction. ${ }^{4}$ Therefore, we speculate that the mid-pregnancy fall has certain clinical implications. By contrast, Silva et al. reported that there was no midpregnancy fall in BP. ${ }^{5}$ Their study was a part of the Generation R Study conducted from 2002 to 2006, a population-based prospective cohort study of subjects from fetal life until young adulthood, with 9778 mothers of various ethnicities and their children in the Netherlands. In the Generation R Study, a mid-pregnancy fall in systolic BP was not found, and the authors discussed the absence of a mid-pregnancy fall in diastolic BP only for women with a low educational level. Thus, whether a mid-pregnancy fall occurs is still uncertain, and further studies must be done to obtain more definitive evidence. Therefore, we are very interested in BP changes during pregnancy among pregnant women with or without hypertensive disorders and among all participants in the study conducted by Jwa et al.

We have also shown that a seasonal trend occurs in BP changes during pregnancy and that the mid-pregnancy fall using home BP measurement is affected by the expected dates of confinement. ${ }^{6}$ In our study, pregnant women who delivered in winter tended to have higher BPs than those who delivered in summer. This finding indicates that the lowest daily temperature affects the BP changes during pregnancy and may contribute to the occurrence of PIH. We are also curious to understand how seasonal effects contribute to predicting $\mathrm{PIH}$ by a shift in BP class in the study by Jwa et al.

It might be difficult to identify small changes, such as a mid-pregnancy fall or seasonal variation, when using clinic BP, because clinic BP has a greater variation than home BP. Macdonald-Wallis et al. showed a clinic BP change during pregnancy in around 10000 normotensive pregnant women in the UK who joined the Avon Longitudinal Study of Parents and Children. ${ }^{7}$ It seems that clinic BPs of normotensive pregnant women had a mid-pregnancy fall, as observed in our study.

Because, in Japan, pregnant women generally visit antenatal care clinics or hospitals once every 4 weeks until week 23 , once every 2 weeks from weeks 24-35, and once a week after week 36 , Jwa et al. might be able to evaluate clinic BP changes during pregnancy in more detail than have previous studies. ${ }^{5,7}$ A detailed evaluation of clinic BP would be helpful for gaining a better understanding of the mechanism of BP changes during pregnancy and for predicting the risk of hypertension during pregnancy. Therefore, we would like to ask Jwa et al. to provide the clinic BP values at each antenatal care visit throughout pregnancy.

\section{CONFLICT OF INTEREST}

Metoki $\mathrm{H}$ is carrying out collaborative research, in addition to the present study, with Omron Healthcare Ltd.

\section{ACKNOWLEDGEMENTS}

This work was supported by Grants for Scientific Research (nos. 18590587, 18390192, 21390201,22890017, and 23590771) from the Ministry of Education, Culture, Sports, Science and Technology of Japan; Grant-in-Aid (H21Junkankitou[Seishuu]- Ippan-004) from the Ministry of Health, Labor and Welfare, Health and Labor Sciences Research Grants, Japan; Grant-inAid form the Japan Society for the Promotion of Science (JSPS) fellows (19.7152, 20.7198,20.7477 and 20.54043); Grants from the Takeda Science Foundation; and Grants from the OTC SelfMedication Promotion Foundation.

Mami Ishikuro $^{1,2}$, Taku Obara ${ }^{1,2}$, Hirohito Metoki ${ }^{2,3}$, Takayoshi Ohkubo ${ }^{4,5}$, Nobuo Yaegashi ${ }^{2,3}$, Shinichi Kuriyama ${ }^{1,2}$ and Yutaka Imai ${ }^{4}$

${ }^{1}$ Division of Molecular Epidemiology, Tohoku University Graduate School of Medicine, Sendai, Japan; ${ }^{2}$ Environment and Genome Research Center; Tohoku University Graduate School of Medicine, Sendai, Japan; ${ }^{3}$ Department of Obstetrics and Gynecology,

Tohoku University Graduate School of Medicine, Sendai, Japan; ${ }^{4}$ Department of Planning for Drug Development and Clinical Evaluation, Tohoku University Graduate School of Pharmaceutical Sciences, Sendai, Japan and 5 Department of Health Science, Shiga University of Medical Science, Otsu, Japan

E-mail: hmetoki@med.tohoku.ac.jp.

1 Jwa SC, Arata N, Sakamoto N, Watanabe N, Aoki H, Kurauchi-Mito A, Dongmei $Q$, Ohya $Y$, Ichihara A, Kitagawa M. Prediction of pregnancy-induced hypertension by a shift of blood pressure class according to the JSH 2009 guidelines. Hypertens Res 2011; 34: 1203-1208.

2 Metoki H, Ohkubo T, Sato Y, Kawaguchi M, Nishimura M, Watanabe Y, Imai Y. Detection of midpregnancy fall in blood pressure by out-of-office monitoring. Hypertension 2009; 53: 12-13.

3 Duvekot JJ, Cheriex EC, Pieters FA, Menheere PP, Peeters LH. Early pregnancy changes in hemodynamics and volume homeostasis are consecutive adjustments triggered by a primary fall in systemic vascular tone. Am J Obstet Gynecol 1993; 169: 1382-1392. 
4 Savvidou MD, Hingorani AD, Tsikas D, Frölich JC, Vallance P, Nicolaides KH. Endothelial dysfunction and raised plasma concentrations of asymmetric dimethylarginine in pregnant women who subsequently develop pre-eclampsia. Lancet 2003; 361: 1511-1517.

5 Silva LM, Steegers EA, Burdorf A, Jaddoe VW, Arends LR, Hofman A, Mackenbach JP, Raat H. No midpregnancy fall in diastolic blood pressure in women with a low educational level: the Generation R Study. Hypertension 2008; 52: 645-651.

6 Metoki H, Ohkubo T, Watanabe Y, Nishimura M, Sato Y, Kawaguchi M, Hara A, Hirose T, Obara T, Asayama K, Kikuya M, Yagihashi K, Matsubara Y, Okamura K, Mori S, Suzuki M, Imai YBOSHI Study Group. Seasonal trends of blood pressure during pregnancy in Japan: the Babies and their Parents' longitudinal observation in
Suzuki Memorial Hospital in intrauterine period study $J$ Hypertens 2008; 26: 2406-2413.

7 Macdonald-Wallis C, Tilling K, Fraser A, Nelson SM, Lawlor DA. Established preeclampsia risk factors are related to patterns of blood pressure change in normal term pregnancy: findings from the Avon Longitudina Study of Parents and Children. J Hypertens 2011; 29 1703-1711. 